über die seinerzeit mit dem Argument der Arbeitsfähigkeit vereinbarte Größe von $600^{47}$ hinaus dann gut begründbar, wenn sie diesen Prinzipien dient.

Nun ist es um das Wahlrecht im Bundestag erst einmal ruhig geworden. Aber, in den Worten von Bundestagspräsident Norbert Lammert in seiner jüngsten Antrittsrede: „[D]a es immer besser ist, sich mit solchen Entwicklungen dann auseinanderzusetzen, wenn die Probleme noch nicht eingetreten sind, spricht manches dafür, dass wir nicht erst nach der nächsten Wahl, sondern rechtzeitig vor der nächsten Wahl noch einmal einen gemeinsamen sorgfältigen Blick auf diese Regelungen werfen. “48

47 Vgl. Deutscher Bundestag, Stenografischer Bericht, 129. Sitzung, Plenarprotokoll 13/129 vom 11. Oktober 1996; Deutscher Bundestag, Zwischenbericht der Reformkommission zur Größe des Deutschen Bundestages, Drs. 13/4560 vom 8. Mai 1996; Deutscher Bundestag, Schlussbericht der Reformkommission zur Größe des Deutschen Bundestages, Drs. 13/7950 vom 17. Juni 1997.

48 Deutscher Bundestag, Stenografischer Bericht, Plenarprotokoll 18/1 vom 22. Oktober 2013, S. 8.

\title{
Erfolgswertgleichheit als Maßstab für die Sitzverteilung in Parlamenten
}

Fred Hermsdorf

In Wahlgesetzen wird unter anderem geregelt, in welcher Form die abgegebenen Stimmen zur Ermittlung der Sitzverteilung im Parlament herangezogen werden und nach welchen Verfahren bei Verhältniswahlen die entsprechenden Stimmen auf die Parteien verteilt werden. Zur Berechnung dieser Sitzverteilung existieren mehrere verschiedene Methoden (Hare/Niemeyer, St.Laguë/Schepers usw.). Dieser Beitrag bezieht sich allein auf diese Berechnungsverfahren. Der weitere Einfluss der Wahlgesetze auf die zu verteilenden Parlamentssitze wird außer Acht gelassen.

Es ist im Allgemeinen nicht eindeutig möglich, die (große) Anzahl der Wählerstimmen auf die (kleine) Anzahl der Sitze im Parlament proportional abzubilden. Da es keine Teile von Abgeordneten gibt, entsteht ein Spannungsfeld zwischen exakter Proportionalität und Ganzzahligkeit. Es sind politische Entscheidungen zu treffen, wie mit den (zwangsläufig) überraschenden Effekten der berechneten Sitzverteilungen umzugehen ist. Natürlich sind bei allen diesen Entscheidungen die Vorgaben der Verfassung zu beachten.

Die Auswahl des geeigneten Verfahrens hängt somit von den besonderen Anforderungen $\mathrm{ab}$, die in dem betreffenden Wahlgebiet gelten. Liest man etwa in dem bekannten Werk von Balinski und Young ${ }^{1}$, so wird deutlich, dass dort die Überlegungen zur Überwindung dieser Effekte sehr stark geprägt sind von den Bedingungen der Vereinigten Staaten von Amerika im 19. Jahrhundert, vor allem von Bevölkerungswachstum und dem Entstehen neuer Bundesstaaten.

1 Vgl. Michael L. Balinski / H. Peyton Young, Fair Representation - Meeting the Ideal of One Man, One Vote, New Haven / London 1982. 
In der Bundesrepublik Deutschland ist durch das Bundesverfassungsgericht festgelegt, dass die Erfolgswertgleichheit der Wählerstimmen als Maßstab anzuwenden ist. Da die exakte Gleichheit kaum zu erreichen ist, sollte zumindest das letztlich angewendete Berechnungsverfahren die Erfolgswertunterschiede so gering wie möglich halten.

Bei der Konstruktion der gegenwärtig zur Auswahl stehenden Verfahren spielte der Erfolgswert aber keine Rolle. ${ }^{2}$ Ihr Verhalten bezüglich des Erfolgswertes wurde erst nachträglich untersucht. Es stellt sich somit die Frage, warum in der Bundesrepublik nicht ein Berechnungsverfahren Anwendung findet, das sich von Anfang an allein auf den Erfolgswert konzentriert. Dass eventuell keine einfache Berechnung zum Ziel führt, kann kein Hinderungsgrund sein, denn durch Computer steht Rechenkapazität im großen Umfang zur Verfügung. Auch beim Berechnungsverfahren des am 21. Februar 2013 verabschiedeten neuen Wahlgesetzes ist der Rechenaufwand nicht unerheblich. ${ }^{3}$

Wie sich zeigen wird, ergeben sich bei einem Verteilungsverfahren, das den Erfolgswert als primären Ansatz verwendet, wie auch bei allen anderen Verfahren gewisse überraschende Verteilungen. Proportionalität und Ganzzahligkeit stehen eben auch hier in Konkurrenz. Von diesen auch Paradoxien genannten Effekten sollen hier nur zwei angeführt werden.

(1) Die „unlogischen “Sprünge (die Wortwahl suggeriert, dass diese Erscheinung negativ ist): Nicht gesagt wird dabei, welche Logik gemeint ist. Diese Erscheinung besagt, dass bei Erhöhung der Sitzanzahl im Parlament eine Partei einen Sitz verlieren kann. Bei Problemen im Bereich der ganzen Zahlen können aber schon kleine Abweichungen bei den Randbedingungen zu Sprüngen im Ergebnis führen. So überraschend oder sogar unlogisch ist diese Erscheinung somit nicht. Die erste Entdeckung dieser Eigenschaft wurde dann auch weniger spektakulär als Alabama-Paradoxon benannt, weil es in den USA bei der Zuteilung der Parlamentssitze für den Bundesstaat Alabama auffiel.

(2) Der Umkehreffekt: Es ist möglich, dass ein Berechnungsverfahren einem Zusammenschluss von Parteien, die zusammen die Mehrheit der Wählerstimmen erhalten haben, im Parlament die Mehrheit an Sitzen verweigert. Diesem schwerwiegenden Problem wird im Gegensatz zu den „unlogischen“ Sprüngen keine allzu große Aufmerksamkeit geschenkt. Dass es aber doch nicht ganz unbemerkt geblieben ist, kann man im Gesetzentwurf der Fraktionen CDU/CSU, FDP, SPD und Bündnis 90/Die Grünen von 2013 sehen. ${ }^{4}$ Allerdings bleibt man hier bei dem bisher noch nie in der Bundesrepublik aufgetretenen Fall stehen, dass eine einzige Partei die Mehrheit der Wählerstimmen erreicht aber nicht die Mehrheit der Sitze im Parlament. Warum die höchst relevante Frage von möglichen Koalitionen nicht aufgegriffen wird, ist nicht ersichtlich.

2 Die Fraktionen von CDU/CSU, SPD, FDP und Bündnis 90/Die Grünen einigten sich mit einem gemeinsamen Gesetzentwurf darauf die Bundestagswahl 2013 nach einem neuen Wahlrecht abzuhalten. Nach einer Expertenanhörung empfahl der Innenausschuss am 20. Februar 2013 dem Plenum die Annahme des Entwurfs. Einen Tag später beschloss der Bundestag die Novellierung des Bundeswahlgesetzes gegen die Stimmen der Fraktion Die Linke. Vgl. Nils Dehmel / Eckhard Jesse, Das neue Wahlgesetz zur Bundestagswahl 2013. Eine Reform der Reform ist unvermeidlich, in: ZParl, 44. Jg. (2013), H. 1, S. 201 - 213; Joachim Behnke, Das neue Wahlgesetz im Test der Bundestagswahl 2013, in: ZParl, 45. Jg. (2014), H. 1, S. 17 - 37.

3 Für das Berechnungsverfahren vgl. Deutscher Bundestag, Beschlussempfehlung und Bericht des Innenausschusses (4. Ausschuss), BT-Drs. 17/12417 vom 20. Februar 2013, S. 4 f.

4 Vgl. ebenda, $\$ 7$ Sonderfälle (3), S. 5. 
Bei diesen beiden Problemen sollte immer berücksichtigt werden, dass die Verfahren konstruiert wurden, um vorrangig dem Wählerwillen gerecht zu werden. Dass sich für einzelne Parteien unter Umständen Nachteile ergeben können, muss als Folge dieser Priorität in Kauf genommen werden.

Neben den als unschön betrachteten Effekten gibt es aber auch eine positive Eigenschaft, die immer wieder Erwähnung findet: die Einhaltung der Quote. Darunter ist folgendes zu verstehen: Berechnet man die exakte Sitzverteilung für eine bestimmte Partei, so ergeben sich im Allgemeinen Nachkommastellen. Teilt ein Berechnungsverfahren allen Parteien eine Sitzzahl zu, die der Vorkommastelle oder der Vorkommastelle +1 entspricht, dann spricht man von Einhaltung der Quote (beste ganzzahlige Annäherung an die Proportionalität).

Wie oben schon angeführt, wurden die gängigen Berechnungsverfahren erst nachträglich auf ihren Bezug zum Erfolgswert untersucht. Folgendermaßen wird dabei vorgegangen:

Hat eine Partei i $v_{i}$ Stimmen und $m_{i}$ Sitze erhalten und ist V die Summe aller Stimmen und $\mathrm{M}$ die Gesamtzahl der Sitze im Parlament, dann ist der Erfolgswert dieser Partei gegeben durch den Ausdruck

$$
\left(m_{i} / M\right) /\left(v_{i} / V\right)
$$

$\left(m_{i} / M\right)$ ist dabei der Anteil der der Partei i zugeteilten Sitze an der Gesamtzahl aller zu vergebenden Sitze und $\left(v_{i} / V\right)$ der Anteil der von der Partei i gewonnenen Stimmen an der Gesamtzahl aller abgegeben Stimmen. Setzt man diese beiden Werte ins Verhältnis zueinander, so ergibt sich ein Vergleichswert für alle Parteien untereinander. Im Idealfall ist der Wert für alle Parteien gleich 1.

Betrachtet man n Parteien, so erhält man für jede Sitzverteilung n Werte. Um die bei den unterschiedlichen Berechnungsmethoden ermittelten Sitzverteilungen miteinander vergleichbar zu machen, wird aus den n Erfolgswerten einer Sitzverteilung ein einziger Wert berechnet, um so die „beste“ Methode zu bestimmen. Für die Berechnung dieses Wertes kommen mehrere Möglichkeiten in Frage, von denen einige im Folgenden betrachtet werden.

\section{Verfahrensbewertung durch Abweichung vom Idealwert}

Bei dieser Betrachtung rückt die Abweichung vom Idealwert in den Mittelpunkt, wobei der kleinste berechnete Wert die beste Sitzverteilung bestimmt.

Zuerst betrachten wir den Fall, bei dem die Summe der Quadrate der Abweichungen vom Idealwert den Vergleichswert ergibt, das heißt

$$
\begin{gathered}
f_{1}(m)=v_{1} *\left(\left(m_{1} / M\right) /\left(v_{1} / V\right)-1\right)^{2}+\ldots+v_{n} *\left(\left(m_{n} / M\right) /\left(v_{n} / V\right)-1\right)^{2} \\
m=\left(\begin{array}{lll}
m_{1}, & \ldots & \left., m_{n}\right)
\end{array}\right) \text { umfasst alle möglichen Sitzverteilungen. }
\end{gathered}
$$

Hier gilt: Lieber die einfache Abweichung zweimal akzeptieren als die doppelte einmal. In gewissem Sinn werden dadurch Ausreißer vermieden. Bewiesen ist, dass eine nach St. Laguë/Schepers berechnete Sitzverteilung diesen Vergleichswert zum Minimum macht. Bei diesem Verfahren gibt es keine „unlogischen“ Sprünge; das Auftreten des Umkehreffektes und die Einhaltung der Quote ist nicht garantiert. 
Als zweite Möglichkeit kommt als Vergleichswert die größte Abweichung vom Idealwert in Frage.

$$
\begin{aligned}
& f_{2}(m)=\max \left|v_{i} *\left(\left(m_{i} / M\right) /\left(v_{i} / V\right)-1\right)\right|(1 \leq i \leq n) \\
m & =\left(m_{1}, \ldots, m_{n}\right) \text { umfasst alle möglichen Sitzverteilungen } .
\end{aligned}
$$

Eine nach Hare/Niemeyer berechnete Sitzverteilung macht diesen Vergleichswert ebenfalls zum Minimum. Da dieses Verfahren auch die Tendenz zeigt, bei größeren Sitzzahlen den Umkehreffekt zu vermeiden ${ }^{5}$, kann man als Pluspunkte für diese Methode anführen:

- Einhaltung der Quote,

- minimale Abweichung des Erfolgswertes vom Idealwert,

- bei „größeren“ Parlamenten kein Umkehreffekt.

Die Vermeidung der „unlogischen“ Sprünge dagegen ist nicht möglich.

In den Tabellen 1 und 2 werden, beruhend auf den Bundestagswahlergebnissen von 1998, die Werte von $f_{2}(m)$ für die nach Hare/Niemeyer und nach St.Laguë/Schepers berechneten Sitzverteilungen zum Vergleich dargestellt.

\begin{tabular}{|l|c|c|c|c|c|}
\hline \multicolumn{6}{|l|}{ Tabelle 1: Verfahren von Hare/Niemeyer } \\
\hline & Stimmen & 655 Sitze & 656 Sitze & 657 Sitze & 658 Sitze \\
\hline SPD & 20.181 .269 & 285 & 285 & 286 & 286 \\
CDU & 14.004 .908 & 198 & 198 & 198 & 198 \\
CSU & 3.324 .480 & 47 & 47 & 47 & 47 \\
Grüne & 3.301 .624 & 47 & 47 & 47 & 47 \\
FDP & 3.080 .955 & 43 & 43 & 44 & 44 \\
PDS & 2.515 .454 & 35 & 36 & 35 & 36 \\
\hline$f_{2}(m)$ & 35600,336 & 38921,966 & 43194,358 & 39983,046 \\
\hline Quelle: Eigenen Berechnungen; Wahlergebnisse 1998: Bundeswahlleiter. \\
\hline
\end{tabular}

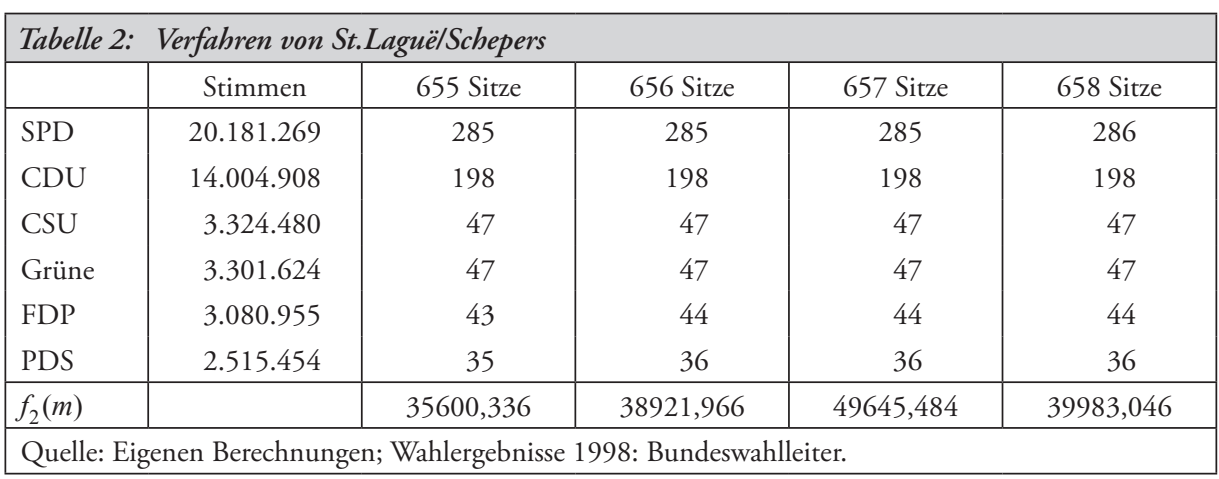

5 Vgl. Fred Hermsdorf, Demokratieprinzip versus Erfolgswertgleichheit. Verfahren der Mehrheitstreue bei Parlamentswahlen, in: ZParl, 40. Jg. (2009), H. 1, S. 86 - 95. 
Wie man sieht, unterscheiden sich die Verteilungen nur bei 657 Sitzen. Hier tritt bei Harel Niemeyer ein „unlogischer“ Sprung auf, gleichzeitig ist dieser aber $f_{2}(m)$ kleiner als bei St.Laguë/Schepers.

\section{Erfolgswertgleichheit}

Das Bundesverfassungsgericht fordert nun aber in seinen Entscheidungen, die Erfolgswertgleichheit und nicht die Abweichung vom Idealwert zu berücksichtigen, das heißt, es schreibt neben der Beachtung des Erfolgswertes zur Beurteilung der Berechnungsmethoden auch die genaue Art und Weise vor, wie der Erfolgswert in die Berechnung der Verfahrensgüte einzubeziehen ist.

Die Betrachtung der Abweichung vom Idealwert kann somit nur als Hilfsweg angesehen werden. Die Gleichheit der Erfolgswerte kann aber wegen des Ganzzahligkeitsproblems nur in Ausnahmefällen erreicht werden, wohl kann man allerdings erwarten, dass die Sitzverteilung gefunden wird, die den größten Unterschied der einzelnen Erfolgswerte so klein wie möglich macht. Es sind somit folgende Werte zu untersuchen:

$$
\left|\left(m_{i} / M\right) /\left(v_{i} / V\right)-\left(m_{j} / M\right) /\left(v_{j} / V\right)\right|(1 \leq i, j \leq n),
$$

das heißt die (positive) Differenz aller möglichen Paare von Erfolgswerten. Wenn man n Parteien betrachtet, entstehen also $\frac{n^{*}(n-1)}{2}$ verschiedene Paare - bei drei Parteien also drei, bei vier Parteien sechs und bei fünf Parteien zehn verschiedene Paare. Insgesamt ist die Anzahl somit überschaubar.

Nach einer einfachen Umformung (Ausklammern von $V / M$ ) erhält man

$$
(V / M) *\left|m_{i} / v_{i}-m_{j} / v_{j}\right|(1 \leq i, j \leq n) \text {. }
$$

Da $V / M$ ein für alle Paare gleicher Faktor ist, muss bei der Ermittlung einer Sitzverteilung folgende Aufgabe gelöst werden: Berechne die Werte $m_{1}$ bis $m_{n}$, die

$$
\max \left|m_{i} / v_{i}-m_{j} / v_{j}\right|(1 \leq i, j \leq n) \text { minimieren. }
$$

Entsprechend der obigen Vorgehensweise wird für jede Sitzverteilung $m_{1}, \ldots, m_{n}$

$$
f_{3}(m)=(V / M) * \max \left|m_{i} / v_{i}-m_{j} / v_{j}\right|(1 \leq i, j \leq n)
$$

als Vergleichswert zur Bestimmung der „besten“ Sitzverteilung herangezogen.

Das Verhalten bezüglich der bekannten Effekte soll an jeweils einem Beispiel erläutert werden, bei dem die angegebene Sitzverteilung $f_{3}(m)$ minimiert wird. 
Anzahl der Sitze: 21

\begin{tabular}{|l|c|c|c|c|c|}
\hline Tabelle 3: Minimierung der maximalen Erfolgswertdifferenzen und die Quote \\
\hline Stimmenzahl & 236 & 235 & 49 & 48 & 48 \\
\hline Vorkommastelle (s.o.) & 8 & 8 & 1 & 1 & 1 \\
Sitze & 8 & 7 & 2 & 2 & 2 \\
\hline Quelle: Eigene Berechnungen.
\end{tabular}

Die berechnete Sitzverteilung liegt für die zweite Partei nicht innerhalb der Quote.

Anzahl der Sitze: 23

\begin{tabular}{|l|c|c|c|}
\hline \multicolumn{4}{|l|}{ Tabelle 4: Minimierung der maximalen Erfolgswertdifferenzen und der Umkehreffekt } \\
\hline Stimmenzahl & 7.805 & 4.538 & 3.117 \\
\hline Prozentanteil & 50,485 & 29,353 & 20,162 \\
Sitze & 11 & 7 & 5 \\
\hline Quelle: Eigene Berechnungen. & \multicolumn{4}{|l}{} \\
\hline
\end{tabular}

Die erste Partei hat trotz 50,484 Prozent der Stimmen nur elf von 23 Sitzen.

\begin{tabular}{|l|c|c|c|}
\hline \multicolumn{4}{|c|}{ Tabelle 5: Minimierung der maximalen Erfolgswertdifferenzen und die „unlogischen “Sprünge } \\
\hline Stimmenzahl & 7.805 & 4.538 & 3.117 \\
\hline 22 Sitze & 12 & 6 & 4 \\
23 Sitze & 11 & 7 & 5 \\
\hline Quelle: Eigene Berechnungen.
\end{tabular}

Die erste Partei verliert einen Sitz beim Übergang von 22 auf 23 Sitze.

Aus diesen drei Beispielrechnungen wird ersichtlich, dass die Forderung nach der Erfolgswertgleichheit beziehungsweise ihrer besten Erfüllung weder die Einhaltung der Quote noch die Vermeidung des Umkehreffektes sowie der „unlogischen“ Sprünge garantieren kann.

In Tabelle 6 werden die Sitzverteilungen dargestellt (am Beispiel der Bundestagswahl 1998), die sich bei dem aus der Forderung nach Erfolgswertgleichheit abgeleiteten Berech-

\begin{tabular}{|c|c|c|c|c|c|}
\hline \multirow{2}{*}{ Tabelle 6: } & \multicolumn{5}{|c|}{ Verfahren der Minimierung der maximalen Erfolgswertdifferenzen } \\
\hline & Stimmen & 655 Sitze & 656 Sitze & 657 Sitze & 658 Sitze \\
\hline SPD & 20.181 .269 & 284 & 284 & 285 & 286 \\
\hline $\mathrm{CDU}$ & 14.004 .908 & 197 & 198 & 198 & 198 \\
\hline CSU & 3.324 .480 & 47 & 47 & 47 & 47 \\
\hline Grüne & 3.301 .624 & 47 & 47 & 47 & 47 \\
\hline FDP & 3.080 .955 & 44 & 44 & 44 & 44 \\
\hline PDS & 2.515 .454 & 36 & 36 & 36 & 36 \\
\hline$f_{3}(m)$ & & 0,0174 & 0,0169 & 0,0134 & 0,0123 \\
\hline
\end{tabular}


nungsverfahren ergeben, das heißt genauer gesagt der „Minimierung der maximalen Erfolgswertdifferenzen".

Gegenüber der Verteilung nach Hare/Niemeyer (Tabelle 1) treten Abweichungen bei 655, 656 und 657 Sitzen auf; gegenüber dem Verfahren nach St.Laguë/Schepers (Tabelle 2) ergeben sich Abweichungen bei 655 und 656 Sitzen. Bei letzterem gilt, dass der Austausch einer einzelnen Stimme - etwa zwischen den Parteien i und j- den Wert

$\left|\left(m_{i} / M\right) /\left(v_{i} / V\right)-\left(m_{j} / M\right) /\left(v_{j} / V\right)\right|$

vergrößert. Somit stellt die Verteilung nach St.Laguë/Schepers ein so genanntes lokales Minimum der Erfolgswertdifferenzen bereit. Dies ist sicher eine interessante Eigenschaft des Verfahrens, aber kaum ausreichend, um die Anforderung des Bundesverfassungsgerichtes zu erfüllen. Die Unterschiede in den Tabellen 2 und 6 zeigen nämlich, dass das globale Minimum der Erfolgswertdifferenzen vom lokalen abweicht, wobei die Sitzverteilung die $f_{3}(m)$ über alle Erfolgswertpaare minimiert als globales Minimum bezeichnet werden kann.

In Tabelle 7 wird aufgezeigt, dass es durchaus mehrere Sitzverteilungen geben kann, die von der Verteilung nach St.Laguë/Schepers abweichen und kleinere Werte von $f_{3}(m)$ als diese besitzen.

Mit den Stimmenverhältnissen von Tabelle 2 (und 6) werden Verteilungen für 656 Sitze angegeben.

\begin{tabular}{l}
\hline Tabelle 7: Sitzverteilungen mit maximalen Erfolgswertdifferenzen kleiner als bei St.Laguël \\
Schepers \\
\hline
\end{tabular}

\section{Fazit: Erfolgswertgleichheit als Maßstab muss überdacht oder Verfahren geändert werden}

Es existieren verschiedene Verfahren zur Berechnung von Sitzverteilungen nach einem Wahlvorgang. Ideal wäre eine proportionale Abbildung der großen Menge der Wählerstimmen auf die kleinere Anzahl der Mitglieder im Parlament. Im Allgemeinen treten aber immer Nachkommastellen bei der Ermittlung dieser Proportionalität auf, Bruchteile von Abgeordneten sind aber nicht möglich. Das hat zur Folge, dass bei jedem Berechnungsverfahren Effekte auftreten, die unerwünscht sind, aber systemimmanent.

Eine verfassungskonforme politische Entscheidung ist notwendig, um ein Berechnungsverfahren auszuwählen. In der Bundesrepublik Deutschland hat das Bundesverfassungsgericht die Erfolgswertgleichheit als Maßstab gesetzt. Wegen der Ganzzahligkeitsproblematik ist diese Gleichheit nicht zu garantieren, wohl aber die beste Annäherung. Die Vorgabe des 
Bundesverfassungsgerichtes führt direkt zu der Forderung, nach einer Bundestagswahl jene Sitzverteilung als Wahlergebnis zu wählen, die das oben aufgeführte $\mathrm{Ma} ß f_{3}(m)$ minimiert.

Zur praktischen Lösung dieses Problems gibt es augenblicklich keine einfache Berechnungsformel, um das Wahlergebnis zu bestimmen; aber mathematische Methoden der (diskreten) Optimierung stehen bereit, um diese Sitzverteilung zu berechnen. Entscheidend ist dabei aber nicht die Art und Weise der mathematischen Berechnungsweise, sondern die Erfüllung der Vorgaben des Gerichtes.

Vergleicht man die Werte in den Tabellen 1, 2 und 6, dann sieht man, dass ein Berechnungsverfahren, das ausschließlich auf der Forderung beruht $f_{3}(m)$ zu minimieren, Ergebnisse liefert, die von denen der bisher gängigen Berechnungsverfahren abweichen können, das heißt die gängigen Berechnungsmethoden erfüllen nicht die Forderungen der Karlsruher Richter.

$\mathrm{Zu}$ hinterfragen ist also, ob bei der Bestimmung des Wahlergebnisses nach Bundestagswahlen zu dem Verfahren „Minimierung der maximalen Erfolgswertdifferenzen“ gewechselt oder die Forderung des Bundesverfassungsgerichtes überdacht werden müsste.

Interessant scheinen - obwohl nicht unmittelbar zum Thema gehörend - die Überlegungen, auf welche Weise die Sitzverteilungen in Parlamentsausschüssen berechnet werden. Hier wird auf gängige Berechnungsmethoden zurückgegriffen, ohne deren Entstehungsgeschichte zu berücksichtigen. Ähnlich wie die Erfolgswertgleichheit bei der Wahl zum Parlament müssten wohl auch hier Anforderungen an das Berechnungsverfahren festgelegt und anschließend ein passendes Verfahren ausgewählt oder gegebenenfalls neu entwickelt werden.

\title{
Alle gleich? Analyse der programmatischen Parteienunterschiede bei Bundestagswahlen auf der Basis des Wahl-O-Mats
}

\author{
Uwe Wagschal und Pascal König
}

Als der Entertainer Stefan Raab am Abend des TV-Duells von Angela Merkel und Peer Steinbrück (1. September 2013) die Kanzlerin fragte, ob sie nach dem Ausfüllen des Wahl-OMats vielleicht SPD wählen würde, unterstellte er damit eine weitgehende inhaltliche Übereinstimmung und Annäherung von CDU/CSU und SPD. Dieser Vorwurf geistert seit Jahrzehnten durch die politikwissenschaftliche Literatur, mit zuerst formuliert von Otto Kirchheimer 1965, der die Volksparteien als kaum mehr unterscheidbare „Allerweltsparteien “ bezeichnete. ${ }^{1}$ In der neueren Zeit wird die parteiliche Alternativlosigkeit vermehrt im Zusammenhang mit dem Begriff der „Postdemokratie“ diskutiert. ${ }^{2}$ In den entsprechenden

1 Otto Kirchheimer, Der Wandel des westeuropäischen Parteiensystems, in: PVS, 6. Jg. (1965), H. 1, S. 20 - 41. So schreibt Kirchheimer: „Sie gibt die Versuche auf, sich die Massen geistig und moralisch einzugliedern, und lenkt ihr Augenmerk in stärkerem Maße auf die Wählerschaft" (S. 27).

2 Siehe zum Beispiel Colin Crouch, Postdemokratie, Frankfurt am Main 2008; Bettina Westle, „Postdemokratien?" - Zur Wahrnehmung der Parteien auf der ideologischen Links-Rechts-Skala: 